\title{
Insulin regulation of solute carrier family 2 member 1 (glucose transporter 1) expression and glucose uptake in decidualizing human endometrial stromal cells: an in vitro study
}

\author{
Ivika Jakson ${ }^{1,2^{*}} \mathbb{D}$, Dorina Ujvari ${ }^{1}$, Sebastian Brusell Gidlöf ${ }^{3,4}$ and Angelica Lindén Hirschberg ${ }^{1,5}$
}

\begin{abstract}
Background: Solute carrier family 2 member 1 (SLC2A1; previously known as glucose transporter 1), is the most abundant glucose transporter in human endometrium and is up-regulated during decidualization, whereas high insulin may have a negative impact on this process. The present study aimed to investigate the effect of insulin on the expression of SLC2A1 and glucose uptake in decidualizing human endometrial stromal cells.

Methods: We induced in vitro decidualization of endometrial stromal cells obtained from regularly menstruating healthy non-obese women. The cells were treated with increasing concentrations of insulin, and the involvement of the transcription factor forkhead box $\mathrm{O} 1$ (FOXO1) was evaluated using a FOXO1 inhibitor. SLC2A1 mRNA levels were measured by Real-Time PCR and protein levels were evaluated by immunocytochemistry. Glucose uptake was estimated by an assay quantifying the cellular uptake of radioactive glucose. One-way ANOVA, Dunnett's multiple comparisons test and paired t-test were used to determine the statistical significance of the results.

Results: We found that insulin dose-dependently decreased SLC2A1 mRNA levels and decreased protein levels of SLC2A1 in decidualizing human endometrial stromal cells. Transcriptional inactivation of FOXO1 seems to explain at least partly the down-regulation of SLC2A1 by insulin. Glucose uptake increased upon decidualization, whereas insulin treatment resulted in a slight inhibition of the glucose uptake, although not significant for all insulin concentrations.

Conclusions: These results indicate an impairment of decidualization by high concentrations of insulin. Future studies will determine the clinical significance of our results for endometrial function and decidualization in women with insulin resistance and hyperinsulinemia.
\end{abstract}

Keywords: Decidualization, GLUT1, SLC2A1, Insulin, Glucose uptake, Human endometrium

\footnotetext{
* Correspondence: ivika.palu@ki.se

'Department of Women's and Children's Health, Karolinska Institutet,

Karolinska vägen 37A, 17176 Stockholm, Sweden

Women's Health Theme, Karolinska University Hospital, Stockholm, Sweden

Full list of author information is available at the end of the article
}

\section{$\triangle B M C$}

C C The Author(s). 2020 Open Access This article is licensed under a Creative Commons Attribution 4.0 International License, which permits use, sharing, adaptation, distribution and reproduction in any medium or format, as long as you give appropriate credit to the original author(s) and the source, provide a link to the Creative Commons licence, and indicate if changes were made. The images or other third party material in this article are included in the article's Creative Commons licence, unless indicated otherwise in a credit line to the material. If material is not included in the article's Creative Commons licence and your intended use is not permitted by statutory regulation or exceeds the permitted use, you will need to obtain permission directly from the copyright holder. To view a copy of this licence, visit http://creativecommons.org/licenses/by/4.0/ The Creative Commons Public Domain Dedication waiver (http://creativecommons.org/publicdomain/zero/1.0/) applies to the data made available in this article, unless otherwise stated in a credit line to the data. 


\section{Background}

Decidualization is a progesterone-induced extensive remodeling of the endometrium in preparation for a potential pregnancy. The extensive remodeling includes morphological transformation of spindle-shaped endometrial stromal cells into decidual cells characterized by increased size and circularity, specific subcellular composition with more ribosomes, lysosomes, glycogen and lipids, and comprehensive reprogramming of the gene expression profile [1, 2]. Improper decidual transformation is proposed to lead to various pregnancy complications, like impaired implantation, recurrent pregnancy loss and preeclampsia [1, 3, 4].

During decidualization, stromal cells show increasing levels of glycogen indicating a glucose-dependent process [5]. Glucose transporters are membrane proteins responsible for glucose uptake in the cells. Their tissue distribution depends on metabolic activity and energy needs of the tissue. At least seven isoforms have been identified in human endometrial stromal cells (hESC). Solute carrier family 2 member 1 (SLC2A1; previously known as glucose transporter 1) is the most abundant glucose transporter in the endometrial stromal cells [6]. In contrast to solute carrier family 2 member 4 (SLC2A4; previously known as GLUT4) that relocates from intracellular vesicles to the plasma membrane only upon insulin signaling, SLC2A1 is classically a non-insulin-responsive glucose transporter responsible for the constitutive transport of glucose. However, insulin has been shown to regulate expression and to some extent cellular location of SLC2A1 in different tissues [7-9].

SLC2A1 protein levels increase up to ten times during decidualization. This increase in SLC2A1 is accompanied by an increase in cellular glucose uptake $[10,11]$. On the other hand, both knockdown of SLC2A1 and in vitro decidualization under low-glucose conditions are associated with decreased decidual marker expression [6, 12]. Together, these data suggest a functional role of SLC2A1 in decidualization.

We have previously demonstrated that insulin affects the expression of many decidual markers via transcriptional and post-translational inhibition of forkhead box O1 (FOXO1), an important transcription factor during decidualization [13]. Our study suggested that high concentrations of insulin may have a negative impact on the comprehensive reprogramming of gene expression during decidualization, However, morphological transformation was not inhibited by insulin. These results could be clinically relevant to hyperinsulinemic conditions like polycystic ovary syndrome (PCOS) and obesity associated with endometrial dysfunction, reduced fertility, recurrent pregnancy loss, preeclampsia and gestational diabetes $[14,15]$. In support, we showed that weight reduction in obese women with PCOS, resulted in enhanced endometrial insulin signaling and increased SLC2A1 expression [16]. This observation led us to the hypothesis that insulin might regulate SLC2A1 during decidualization of human endometrial stromal cells.

There is lack of knowledge about how insulin and SLC2A1 might interact in the glucose-dependent process of decidualization. Further studies of such interaction could increase our understanding of underlying mechanisms for reduced fertility and pregnancy complications in disorders of hyperinsulinemia. The main objective of the present in vitro study was to investigate the effect of different doses of insulin on the gene and protein expression of SLC2A1 in relation to glucose uptake in decidualizing human endometrial stromal cells. Furthermore, we aimed to study if SLC2A1 is regulated by FOXO1.

\section{Methods \\ Subjects}

The subjects were six healthy women aged 21-33 with regular menstrual cycles, body mass index $22-28 \mathrm{~kg} / \mathrm{m}^{2}$, no hormonal treatment 3 months prior to examination, no chronic disease, non-smoking and no regular medication. On cycle day 5-9, an endometrial biopsy was collected using an endometrial suction curette (Pipet Curet, CooperSurgical, USA).

\section{Isolation of endometrial stromal cells}

Endometrial stromal cells were isolated immediately after collection using a protocol previously described [13]. Sequential culturing and immunocytochemistry with cytokeratin and CD10 staining ensured the purity of the obtained stromal cells.

\section{Culture conditions}

We used 6-well Costar plates (Sigma-Aldrich, USA) for all experiments except immunocytochemistry where Falcon chambered cell culture slides (Thermo Fischer Scientific, USA) were used. hESC were cultured until 70$80 \%$ confluence in a culture media consisting of DMEM/ F12-Glutamax (Thermo Fischer Scientific, USA), 10\% fetal bovine serum (Thermo Fischer Scientific, USA) and $0.2 \%$ penicillin-streptomicin (Thermo Fischer Scientific, USA). Cells were then decidualized in phenol-red free DMEM/F12 (Thermo Fischer Scientific, USA) supplemented with $2 \%$ charcoal stripped fetal bovine serum (Sigma-Aldrich, USA) and $0.2 \%$ penicillin-streptomycin for 5-6 days (depending on the experiment) using $1 \mu \mathrm{M}$ medroxyprogesterone-17-acetate (MPA) (Sigma-Aldrich, USA) and $0.5 \mathrm{mM} \mathrm{N}^{6}, 2^{-}$-O-dibutyryladenosine cAMP (db-cAMP) (Sigma-Aldrich, USA) in the presence or absence of 5, 50 or $500 \mathrm{nM}$ insulin (Sigma-Aldrich, USA). The insulin concentrations were chosen based on previous reports of tissue concentrations of insulin in 
different organs being significantly higher than circulating levels. In the experiment with FOXO1 inhibitor, cells were pre-decidualized for 3 days before further treatment with decidualizing agents in the presence of 100 $\mathrm{nM}$ and $500 \mathrm{nM}$ AS1842856 (FOXO1-inhibitor by Merck Millipore, Germany, dissolved in DMSO) for 2 more days. The culture media was changed every 3 days during culturing and treatments.

\section{RNA isolation, CDNA synthesis and RT-PCR}

RNA isolation and cDNA synthesis were done as previously described [16]. The SLC2A1 gene expression level was quantified with Real-Time PCR using TaqMan Assay (Assay ID Hs00892681_m1 for SLC2A1 and Hs01926559_g1 for ribosomal protein L13a as an internal control). All determinations were performed in triplicate and the relative gene expression levels were determined using the $\Delta \Delta \mathrm{C}_{\mathrm{T}}$ method.

\section{Immunocytochemistry}

Cells were treated with $1 \mu \mathrm{M} \mathrm{MPA}$ and $0.5 \mathrm{mM} \mathrm{N}^{6} \mathrm{db}$ cAMP in the presence or absence of $500 \mathrm{nM}$ insulin. After treatment, the cells were fixed with $4 \%$ paraformaldehyde for $15 \mathrm{~min}$ and kept on $4 \mathrm{C}^{\circ}$ with PBS (phosphate-buffered saline) $+0.1 \%$ BSA (bovine serum albumin) before staining. The cells were permeabilized with $0.1 \%$ Triton-X for $15 \mathrm{~min}$, washed several times with PBS and TBS (Tris-buffered saline). After that, the samples were blocked with Background Sniper (Histolab Products AB, Sweden) for $10 \mathrm{~min}$, rinsed with TBS several times and incubated with the primary antibody diluted in DaVinci Green Diluent (Histolab Products AB, Sweden) for $1 \mathrm{~h}$ at room temperature. Two different SLC2A1 antibodies binding to different epitopes were used as primary antibody specificity controls (ab32551 rabbit polyclonal antibody to SLC2A1 from Abcam, dilution 1:200, and LS-C87465 - mouse polyclonal antibody to SLC2A1 from LifeSpan BioSciences, Inc., dilution 1: 400). Secondary antibody and labeling controls were included. After washing with TBS, the samples were incubated with MACH3 Mouse or Rabbit Probe (depending on the primary antibody, Histolab Products AB, Sweden) for $12 \mathrm{~min}$, rinsed with TBS and incubated with MACH3 Mouse or Rabbit M-Polymer HRP for $15 \mathrm{~min}$ in room temperature. The slides were rinsed again with TBS several times before incubation with Betazoid $\mathrm{DAB}\left(3,3^{\prime}\right.$ diaminobenzidine) Chromogen (Histolab Products $A B$, Sweden) for $5 \mathrm{~min}$ and washed with distilled water. The samples were subsequently counter stained with concentrated hematoxylin for $1 \mathrm{~min}$ and rinsed with tap water. Then the slides were dehydrated and mounted with Pertex before examination under microscope.

Samples from all 6 subjects were included in the evaluation. The stained slides were evaluated by three independent evaluators blinded for treatment and sample identity using the semi-quantitative manual scoring on a 5 point-scale: $0(-), 1(+/-), 2(+), 3(++)$ and 4 $(+++)$. Conventional light microscopy at a magnification of 100x was used. Slides were evaluated for staining intensity and percentage of cells with each intensity. Because of the non-homogenic nature of the decidualization process, the four most decidualized areas from each slide were chosen for evaluation and photographed using image analysis system (Leica Imaging System Ltd., Cambridge, UK) before evaluation. The following equation was used to calculate the scores: $4 \mathrm{x}$ percentage of strongly staining cells $+3 \mathrm{x}$ percentage of moderately staining cells $+2 \mathrm{x}$ percentage of weakly staining cells $+1 \mathrm{x}$ percentage of very weakly staining cells, giving a range of 0 to 400 . Scores were expressed as the highest value from the three observers and the highest score for each condition was used in statistical evaluation.

\section{Glucose uptake assay}

Endometrial stromal cells were cultured and treated as described above using decidualizing agents and different concentrations of insulin. Before running the glucose uptake assay, the cells were starved in a serum- and insulin-free media containing $0.5 \mathrm{mM}$ db-cAMP and $1 \mu \mathrm{M}$ MPA for 4 hours. Glucose uptake was estimated by quantifying the cellular uptake of radioactive $[3 \mathrm{H}]$ 2DG (1- $\left[{ }^{3} \mathrm{H}\right]-2$-deoxy-D-glucose). The cells were incubated with [3H]-2DG containing glucose-free DMEM for $15 \mathrm{~min}$, washed, solubilized with sodium dodecyl sulfate (SDS) and frozen over night before measuring the radioactivity of the samples in a liquid scintillation counter. Micro $\mathrm{BCA}^{\mathrm{m}}$ protein assay kit (Thermo Fisher Scientific, USA) was used to quantify the amount of protein in each treatment well and glucose uptake was adjusted accordingly. All experiments were done at least in duplicate.

\section{Statistical analysis}

Normality of data was tested with Shapiro-Wilk normality test. One-way ANOVA and Dunnett's multiple comparisons test were used to analyze the effect of insulin on SLC2A1 mRNA (messenger-RNA). Wilcoxon test was used to analyze the effect of decidualization on glucose uptake because the data was not normally distributed. For immunocytochemistry results we used a paired $\mathrm{t}$-test since the data was normally distributed. A $p$-value of less than 0.05 was considered significant. To analyze the effect of decidualization on SLC2A1 gene expression, the effect of a FOXO inhibitor on $S L C 2 A 1$ gene expression, and the effect of insulin on glucose uptake, we adjusted the values of the control group (stromal cells or decidual cells) to 1 and calculated $95 \%$ confidence 


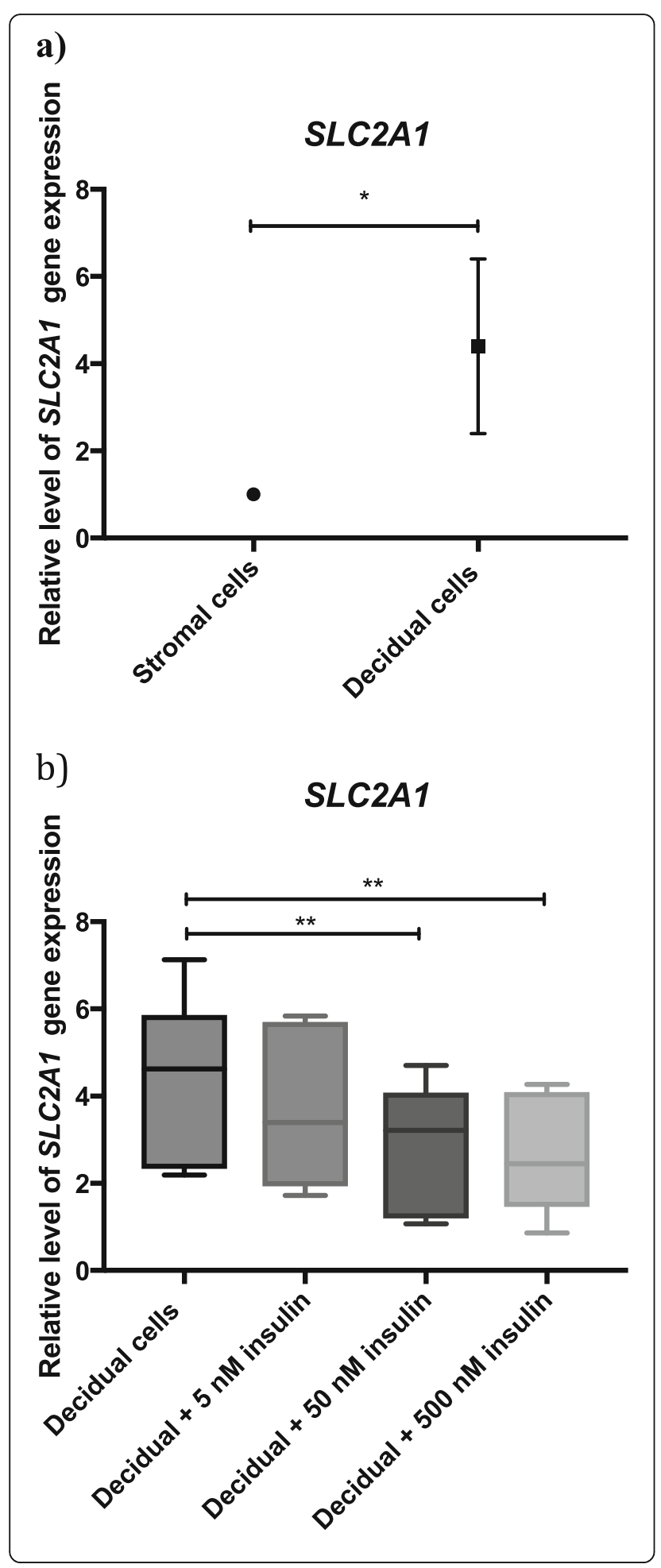

Fig. 1 a Relative gene expression levels of SLC2A1 in human endometrial stromal cells and decidualizing human endometrial stromal cells. The results are presented as mean values with 95\% confidence interval $(\mathrm{Cl})$. Decidualizing endometrial stromal cells were compared to the control group (stromal cells) and differences considered statistically significant if $95 \% \mathrm{Cl}$ did not include the numerical value of the control group, in which case the significance was marked with one asterisk $\left(^{*}\right)$ corresponding to the significance level of $p<0.05$. b Relative gene expression levels of SLC2A1 in response to increasing concentrations of insulin in decidualizing human endometrial stromal cells. The values are presented as box plots showing minimum, first quartile, median, third quartile and maximum. One-way ANOVA and Dunnett's multiple comparisons test was used for statistical analysis. ${ }^{* *}=p<0.01$

intervals $(\mathrm{CI})$ to all other groups. A 95\% CI that did not include the numerical value of the control group was considered statistically significant and marked with one asterisk $\left({ }^{*}\right)$ corresponding to the significance level of $p<0.05$.

\section{Results}

\section{Insulin downregulates SLC2A1 mRNA level in} decidualizing hESC

Both prolactin and insulin like growth factor binding protein 1 (IGFBP-1), two of the most often used decidualization markers, increased significantly upon treating the endometrial stromal cells with decidualizing agents (data not shown). To study the effect of insulin on $S L C 2 A 1$ expression, we decidualized the hESC with and without different concentrations of insulin for 6 days and measured $S L C 2 A 1$ gene expression levels. We confirmed up-regulation of SLC2A1 mRNA and protein expression during decidualization (Fig. 1a and Fig. 3a). Figure $1 \mathrm{~b}$ shows the effect of increasing doses of insulin on the expression of SLC2A1 mRNA. Treatment with $50 \mathrm{nM}$ and $500 \mathrm{nM}$ insulin resulted in significant dosedependent down-regulation of $S L C 2 A 1$ expression, indicating that insulin is a regulator of $S L C 2 A 1$ gene expression at a transcriptional level in decidualizing hESC.

\section{FOXO1 inhibition downregulates the expression of SLC2A1 mRNA in decidualizing hESC}

Insulin transcriptionally inactivates FOXO1 in decidualizing hESC thereby affecting the expression of FOXO1 target genes [13]. To establish whether SLC2A1 mRNA expression is regulated by FOXO1, we decidualized the cells in the presence of a FOXO1 inhibitor. The inhibition of FOXO1 by $100 \mathrm{nM}$ and $500 \mathrm{nM}$ of the inhibitor AS1842856 significantly decreased the expression of SLC2A1 mRNA during decidualization (mean fold change 0.6 and 0.45 , respectively), which indicates that SLC2A1 is regulated by FOXO1 on a transcriptional level (Fig. 2). 


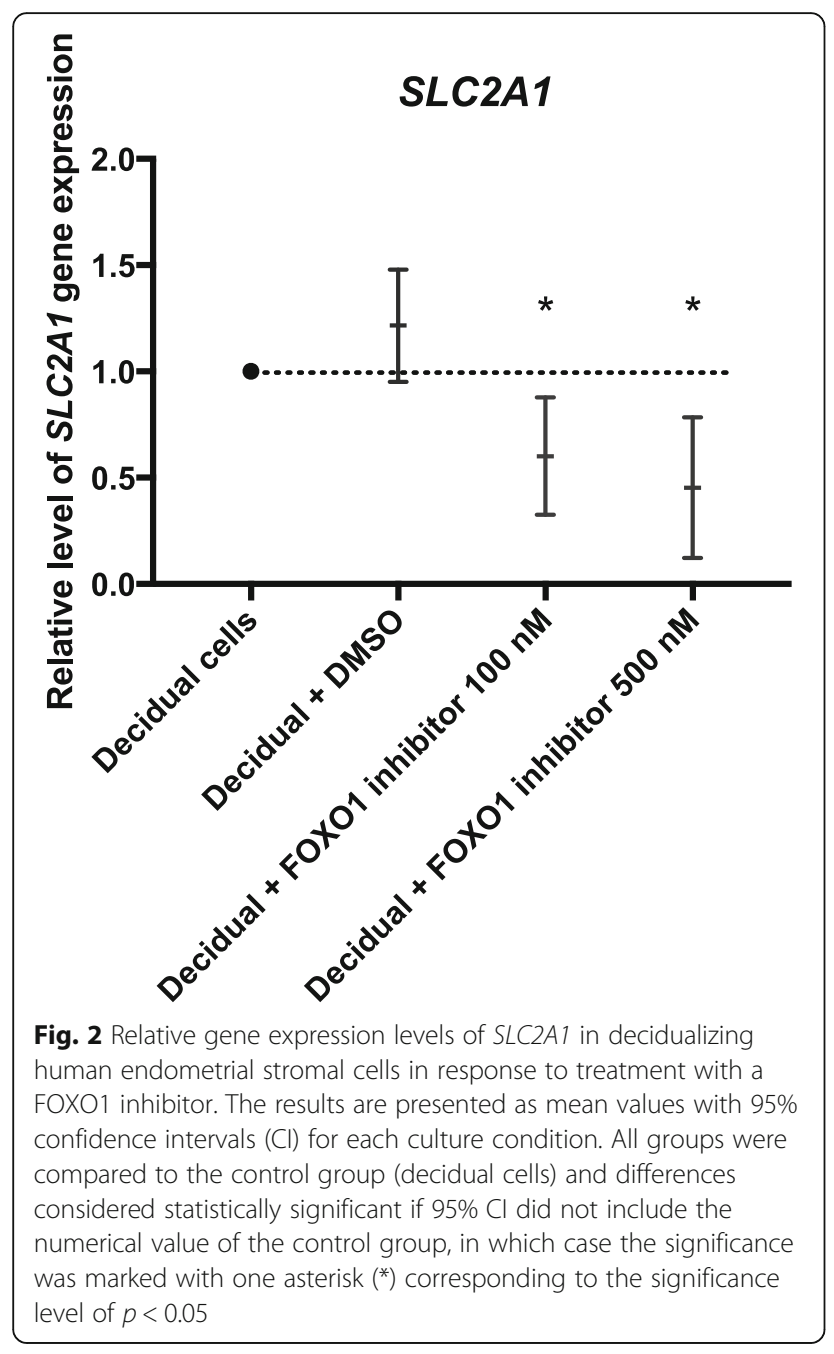

Insulin downregulates SLC2A1 protein level in decidualizing hESC

To evaluate the effect of insulin on SLC2A1 protein expression we decidualized hESC with and without 500 $\mathrm{nM}$ insulin for 6 days and quantified SLC2A1 protein levels using immunocytochemistry (Fig. 3a). Secondary antibody and labeling controls did not show any nonspecific staining. Two different antibodies binding to different epitopes of SLC2A1 protein were used and both resulted in similar staining patterns. We could show a slight but significant down-regulation of SLC2A1 protein expression in insulin-treated cells (Fig. 3a, b).

\section{Insulin decreases glucose uptake in decidualizing hESC}

We measured glucose uptake in both non-decidualized and decidualized endometrial stromal cells and found a significant increase in glucose uptake after decidualization by a median fold change of 4.2 (Fig. 4a). We studied the effect of different concentrations of insulin on glucose uptake in decidualized hESC. In response to insulin, the median level of glucose uptake was lower in all groups but significantly decreased only after treatment with $5 \mathrm{nM}$ insulin (mean fold change 0.69; 95\% CI of 0.46 to 0.93 ) (Fig. 4b).

\section{Discussion}

SLC2A1 expression is upregulated during decidualization, whereas insulin could have a negative impact on the same process. The present study is the first to show that high doses of insulin downregulate SLC2A1 mRNA and protein levels during decidualization of human endometrial stromal cells. The downregulation of SLC2A1 appears to be at least partly mediated by transcriptional inactivation of FOXO1. Decidualization was associated with increased glucose uptake, whereas insulin treatment resulted in a slight inhibition of the glucose uptake, although not significant for all insulin concentrations.

The insulin-mediated glucose uptake by solute carrier family 2 member 4 (SLC2A4:previously known as GLUT4) has been studied in many tissues [17]. However, there is data suggesting that insulin also has an effect on SLC2A1-mediated glucose uptake [8, 18]. SLC2A1 is the most common glucose transporter in human endometrium [6]. We have previously demonstrated that weight loss in obese women with PCOS, resulted in reduced circulating insulin levels, concomitantly enhanced insulin signaling and increased SLC2A1 expression in the endometrium [16]. Furthermore, an in vitro study from our research group suggested that high insulin levels may have a negative impact on the decidual transcriptome by transcriptional inactivation of FOXO1 [13]. We therefore hypothesized that SLC2A1 and insulin might interact in glucose uptake regulation and decidualization of human endometrium.

Indeed, the present study showed that high doses of insulin suppressed the expression of both gene and protein levels of SLC2A1 in decidualizing hESC. To the best of our knowledge, this is the first study on SLC2A1 regulation by insulin in decidualizing endometrial stromal cells derived from experiments using insulin-treated primary endometrial stromal cells. Furthermore, we confirmed for the first time that $S L C 2 A 1$ is a target of FOXO1 [19], an important transcription factor during decidualization. Since insulin is known to affect FOXO1 target genes via transcriptional inhibition [13], the downregulation of SLC2A1 by insulin seems at least partly be mediated by FOXO1.

Decidualization is proposed to be a highly energydependent process resulting in increased cellular glycogen levels [5]. SLC2A1 expression is upregulated and accompanied by an increase in glucose uptake during decidualization [10, 11]. Despite its upregulation upon decidualization, SLC2A1 is not considered as a classical 
a)
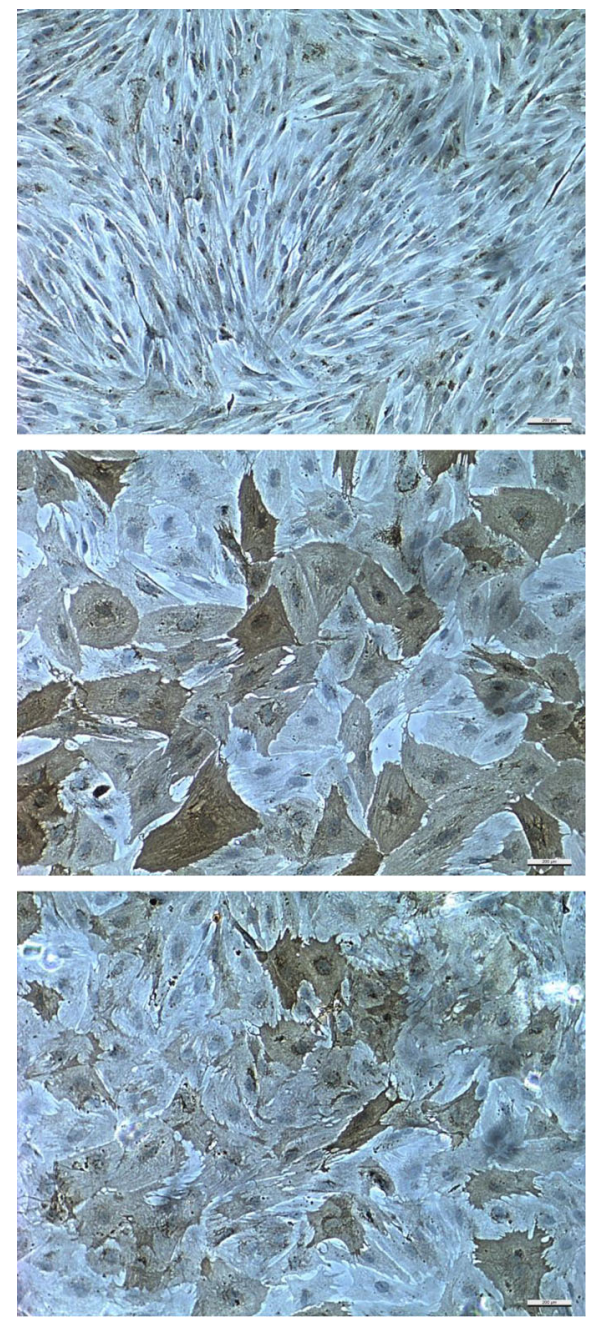

b)

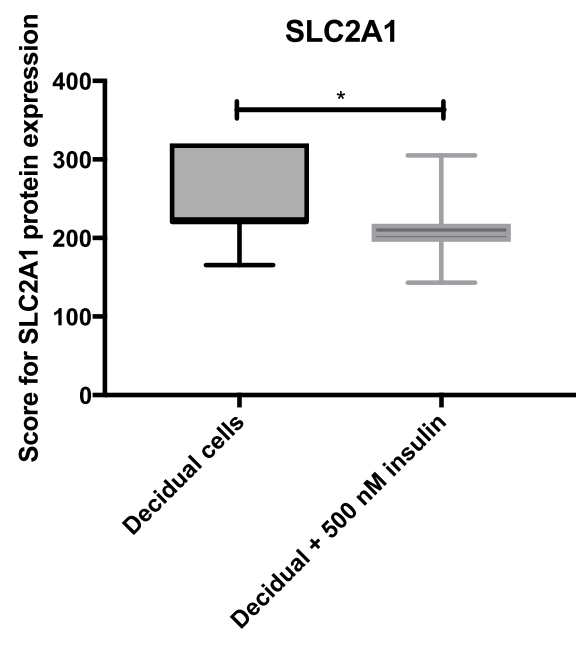

Fig. 3 a Cytoplasmic staining of SLC2A1 protein in human endometrial stromal cells before and after decidualization with and without insulin. Top picture: human endometrial stromal cells, 100 x magnification. Center picture: decidualizing human endometrial stromal cells, $100 \times$ magnification. Bottom picture: human endometrial stromal cells decidualized in the presence of $500 \mathrm{nM}$ insulin, $100 \times$ magnification. $\mathbf{b}$ Relative levels of SLC2A1 protein expression in response to insulin treatment. The values are presented as box plots showing minimum, first quartile, median, third quartile and maximum. Paired t-test was used for statistical analysis. $p<0.05$ was considered significant. ${ }^{*}=p<0.05$

marker of decidualization as it is abundantly expressed in undifferentiated stromal cells, too. Here, we confirm that glucose uptake increases upon decidualization of human endometrial stromal cells [11]. Since SLC2A1 is the dominating glucose transporter in endometrial stromal cells [12], we expected that decreased SLC2A1 expression in response to insulin would result in impaired glucose uptake. In support, we found a slight decrease of glucose uptake by insulin, although not consistent for all insulin concentrations. This is indirect evidence that the effect of insulin on glucose uptake is medicated by SLC2A1. However, we cannot exclude that other glucose transporters also could be involved.
It is unclear how high insulin affects the complex nature of decidualization since it does not seem to affect the morphological changes induced by decidualization. However, we have previously demonstrated extensive changes in the transcription profile of decidualizing endometrial stromal cells by high insulin indicating dysregulation of the process [13]. Furthermore, a recent study by Neff et al. demonstrated that loss of insulin receptor substrate 2 (IRS2), a signaling molecule that mediates the effect of insulin, suppressed the expression of decidualization markers and SLC2A1, and decreased the glucose uptake of the cells. They also showed that high insulin doses caused down-regulation of the insulin 


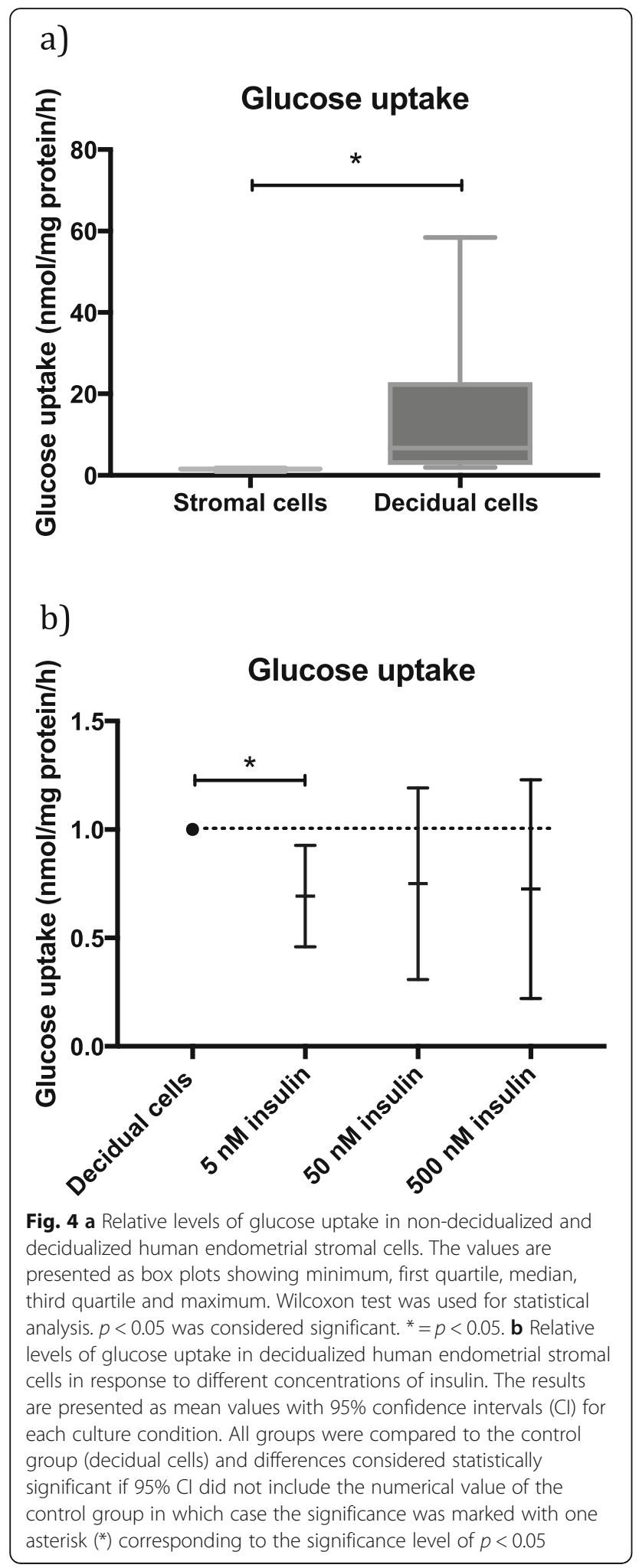

receptor and certain decidualization markers, which is in line with our previous publication $[11,13]$. In the present study, we demonstrate the direct downregulating effect of high doses of insulin on SLC2A1 expression and glucose uptake. These data support the role of insulin in dysregulation of decidualization.

Although the present study is an experimental in vitro investigation, the results may have implications for clinical conditions like PCOS and obesity. These hyperinsulinemic disorders are associated with anovulation but also endometrial dysfunction as reflected by impaired decidualization and implantation, increased risk of miscarriage and preeclampsia [20-22]. On the other hand, lifestyle intervention resulting in weight loss and enhanced insulin sensitivity, improves reproductive function in these women $[16,23]$. Normalized endometrial function and decidualization could be involved in this improvement. Our study provides information on the multifactorial effect of insulin on endometrial decidualization and the regulation of glucose homeostasis. Increased knowledge of molecular mechanisms behind reproductive disorders associated with hyperinsulinemia might lead to new therapeutic approaches in the treatment of infertility.

The limitations of the study include restricted number of endometrial samples and the interindividual variation of the results. The women had regular menstrual cycles but not all had been pregnant and therefore normal fertility was not proven. Also, one of the subjects was overweight with a BMI of 28 . However, we consider the use of primary endometrial stromal cells instead of a cell line a strength of the study. Insulin concentrations were chosen based on previous studies [24-27]. Tissue concentrations of insulin have been shown to vary in different tissues and are not directly related to circulating levels [28]. The normal variation of insulin concentration in the endometrium is not known.

\section{Conclusions}

In conclusion, this study provides a further piece of understanding of the complex process of decidualization. We showed that high concentrations of insulin downregulate both gene and protein expression of SLC2A1 accompanied by a slight decrease in glucose uptake in decidualizing human endometrial stromal cells. Although our results show that SLC2A1 is downregulated by increased insulin, we cannot fully conclude the functional role of SLC2A1 for decidualization as this was not the specific aim of the study. Furthermore, clinical studies are needed to determine the importance of insulin regulation of endometrial function and decidualization in patient groups with insulin resistance and hyperinsulinemia.

\section{Abbreviations}

[3H]-2DG: 1-[ $\left.{ }^{3} \mathrm{H}\right]-2-$ deoxy-D-glucose; BSA: Bovine serum albumin; $\mathrm{Cl}$ : Confidence interval; DAB: 3, 3'-diaminobenzidine; db-cAMP: N6, $2-0$ dibutyryladenosine CAMP; FOXO1: Forkhead box 01; hESC: Human endometrial stromal cells; IRS2: Insulin receptor substrate 2; MPA: Medroxyprogesterone-17-acetate; mRNA: Messenger-RNA; PBS: Phosphate-buffered saline; PCOS: Polycystic ovary syndrome; SDS: Sodium dodecyl sulfate; SLC2A1: Solute carrier family 2 member 1 ; SLC2A4: Solute carrier family 2 member 4; TBS: Tris-buffered saline 


\section{Acknowledgements}

The authors thank Katrin Ingermo (Anna Krook group - Integrative Physiology, Department of Physiology and Pharmacology, Karolinska Institutet) for assistance with the glucose uptake assay, Lotta Blomberg and Berit Legerstam at the Women's Health Clinical Research Unit for technical assistance, and Elena Di Martino and Mariana Paulson for assistance in immunocytochemistry evaluations.

\section{Authors' contributions}

IJ. designed the study, collected the tissue samples, analyzed and interpreted the data and prepared the manuscript. D.U. contributed to the design of the study, interpreted the data and was involved in writing the manuscript. S.B.G. contributed to recruitment of subjects, interpretation of data and writing the manuscript. A.L.H. had overall responsibility for the study, recruited the subjects, collected tissue samples, interpreted the data and contributed to writing the manuscript. The authors read and approved the final manuscript.

\section{Funding}

The research was supported financially by the Swedish Research Council (grant number 2017-02051), Karolinska Institutet, the Stockholm County Council (grant number 20190248) and Åke Wiberg Foundation (grant number M18-0114). The funding bodies have had no role in the design of the study and collection, analysis, and interpretation of data and in writing. Open Access funding provided by Karolinska Institute.

\section{Availability of data and materials}

The datasets used and analyzed during the current study are available from the corresponding author on reasonable request.

\section{Ethics approval and consent to participate}

Informed consent was obtained from all volunteers and the Regional Ethical Committee in Stockholm approved the study (Dnr 2008/865-32 and Dnr 2018/2199-31).

\section{Consent for publication}

Not applicable.

\section{Competing interests}

The authors declare that they have no competing interests.

\section{Author details}

'Department of Women's and Children's Health, Karolinska Institutet, Karolinska vägen 37A, 17176 Stockholm, Sweden. ${ }^{2}$ Women's Health Theme, Karolinska University Hospital, Stockholm, Sweden. ${ }^{3}$ Department of Clinical Science, Intervention and Technology, Karolinska University Hospital, Stockholm, Sweden. ${ }^{4}$ Department of Obstetrics \& Gynecology, Stockholm South General Hospital, Stockholm, Sweden. ${ }^{5}$ Department of Gynecology and Reproductive Medicine, Karolinska University Hospital, Stockholm, Sweden

Received: 29 June 2020 Accepted: 13 November 2020 Published online: 21 November 2020

\section{References}

1. Zhu H, Hou CC, Luo LF, Hu YJ, Yang WX. Endometrial stromal cells and decidualized stromal cells: origins, transformation and functions. Gene. 2014; 551(1):1-14.

2. Pan-Castillo B, Gazze SA, Thomas S, Lucas C, Margarit L, Gonzalez D, et al. Morphophysical dynamics of human endometrial cells during decidualization. Nanomed Nanotechnol Biol Med. 2018;14(7):2235-45.

3. Salker M, Teklenburg G, Molokhia M, Lavery S, Trew G, Aojanepong T, et al. Natural selection of human embryos: impaired decidualization of endometrium disables embryo-maternal interactions and causes recurrent pregnancy loss. PLoS One. 2010;5(4):e10287.

4. Conrad KP, Rabaglino MB, Post Uiterweer ED. Emerging role for dysregulated decidualization in the genesis of preeclampsia. Placenta. 2017;60:119-29.

5. Dean M. Glycogen in the uterus and fallopian tubes is an important source of glucose during early pregnancyt. Biol Reprod. 2019;101(2):297-305.

6. Frolova Al, Moley KH. Glucose transporters in the uterus: an analysis of tissue distribution and proposed physiological roles. Reproduction. 2011; 142(2):211-20
7. Devaskar SU, Mueckler MM. The mammalian glucose transporters. Pediatr Res. 1992;31:1-13.

8. Taha C, Mitsumoto Y, Liu Z, Skolnik EY, Klip A. The insulin-dependent biosynthesis of GLUT1 and GLUT3 glucose transporters in L6 muscle cells is mediated by distinct pathways. Roles of p21(ras) and pp70 S6 kinase. J Biol Chem. 1995;270(42):24678-81.

9. Taha C, Liu Z, Jin J, Al-Hasani H, Sonenberg N, Klip A. Opposite translational control of GLUT1 and GLUT4 glucose transporter mRNAs in response insulin. Role of mammalian target of rapamycin, protein kinase B, and phosphatidylinositol 3kinase in GLUT1 mRNA translation. J Biol Chem. 1999;274(46):33085-91.

10. Frolova A, Flessner L, Chi M, Kim ST, Foyouzi-Yousefi N, Moley KH. Facilitative glucose transporter type 1 is differentially regulated by progesterone and estrogen in murine and human endometrial stromal cells. Endocrinology. 2009;150(3):1512-20.

11. Neff AM, Yu J, Taylor RN, Bagchi IC, Bagchi MK. Insulin signaling via progesterone-regulated insulin receptor substrate 2 is critical for human uterine decidualization. Endocrinol (United States). 2020;161(1):bqz021.

12. Frolova Al, Moley KH. Quantitative analysis of glucose transporter mRNAs in endometrial stromal cells reveals critical role of GLUT1 in uterine receptivity. Endocrinology. 2011;152(5):2123-8.

13. Ujvari D, Jakson I, Babayeva S, Salamon D, Rethi B, Gidlöf S, et al. Dysregulation of in vitro decidualization of human endometrial stromal cells by insulin via transcriptional inhibition of forkhead box protein O1. PLoS One. 2017;12(1):e0171004.

14. Palomba S, De Wilde MA, Falbo A, Koster MPH, Battista G, Sala L, et al. Pregnancy complications in women with polycystic ovary syndrome. Hum Reprod Update. 2015;21(5):575-92.

15. Ke RW. Endocrine basis for recurrent pregnancy loss. Obstet Gynecol Clin North Am. 2014;41(1):103-12.

16. Ujvari D, Hulchiy M, Calaby A, Nybacka A, Byström B, Hirschberg AL. Lifestyle intervention up-regulates gene and protein levels of molecules involved in insulin signaling in the endometrium of overweight/obese women with polycystic ovary syndrome. Hum Reprod. 2014;29(7):1526-35.

17. Petersen MC, Shulman GI. Mechanisms of insulin action and insulin resistance. Physiol Rev. 2018;98(4):2133-223.

18. Sargeant RJ, Pâquet MR. Effect of insulin on the rates of synthesis and degradation of GLUT1 and GLUT4 glucose transporters in 3T3-L1 adipocytes. Biochem J. 1993;290(Pt 3):913-9.

19. Takano M, Lu Z, Goto T, Fusi L, Higham J, Francis J, et al. Transcriptional cross talk between the forkhead transcription factor forkhead box O1A and the progesterone receptor coordinates cell cycle regulation and differentiation in human endometrial stromal cells. Mol Endocrinol. 2007;21(10):2334-49.

20. Talmor A, Dunphy B. Female obesity and infertility. Best Pract Res Clin Obstet Gynaecol. 2015;29(4):498-506.

21. Liu L, Tong X, Jiang L, Li TC, Zhou F, Zhang S. A comparison of the miscarriage rate between women with and without polycystic ovarian syndrome undergoing IVF treatment. Eur J Obstet Gynecol Reprod Biol. 2014;176:178-82.

22. Kjerulff LE, Sanchez-Ramos L, Duffy D. Pregnancy outcomes in women with polycystic ovary syndrome: a metaanalysis. Am J Obstet Gynecol. 2011; 204(6):558.e1-6.

23. Dutton H, Borengasser SJ, Gaudet LM, Barbour LA, Keely EJ. Obesity in pregnancy: optimizing outcomes for mom and baby. Med Clin N Am. 2018; 102(1):87-106

24. Jin Z, Jin Y, Kumar-Mendu S, Degerman E, Groop L, Birnir B. Insulin reduces neuronal excitability by turning on GABAA channels that generate tonic current. PLoS One. 2011;6(1):e16188.

25. Qiao L, Li Y, Sun S. Insulin exacerbates inflammation in fibroblast-like Synoviocytes. Inflammation. 2020;43(3):916-936.

26. Ribeiro M, López de Figueroa P, Blanco FJ, Mendes AF, Caramés B. Insulin decreases autophagy and leads to cartilage degradation. Osteoarthr Cartil. 2016;24(4):731-9.

27. Trujeque-Ramos S, Castillo-Rolón D, Galarraga E, Tapia D, Arenas-López G, Mihailescu S, et al. Insulin regulates GABAA receptor-mediated tonic currents in the prefrontal cortex. Front Neurosci. 2018;12:345.

28. Agardh CD, Lesniak MA, Gerritsen GC, Roth J. The influence of plasma insulin concentrations on tissue insulin levels in rodents: a study of the diabetic chinese hamster and the Ob Ob mouse. Metabolism. 1986;35(3):244-9.

\section{Publisher's Note}

Springer Nature remains neutral with regard to jurisdictional claims in published maps and institutional affiliations. 